INICIATIVAS SUPRANACIONALES ANTE LA CORRUPCIÓN EN EL DEPORTE Y SU INCIDENCIA EN EL ORDENAMIENTO JURÍDICO ESPAÑOL:

EL EJEMPLO DEL CONSEJO DE EUROPA 


\title{
INICIATIVAS SUPRANACIONALES ANTE LA CORRUPCIÓN EN EL DEPORTE Y SU INCIDENCIA EN EL ORDENAMIENTO JURÍDICO ESPAÑOL: EL EJEMPLO DEL CONSEJO DE EUROPA ${ }^{1}$
}

\author{
JAVIER SÁNCHEZ BERNAL ${ }^{2}$ \\ UniversidAd de SALAMANCA, España \\ Prof. de Derecho InTERnacional
}

\section{RESUMEN:}

El deporte ha visto potenciada, en los últimos años, su dimensión económica, dejando de ser únicamente una práctica social saludable. Este hecho ha favorecido la aparición de una nueva criminalidad, caracterizada por el fraude y la corrupción, vinculada a la manipulación de competiciones y a las apuestas deportivas. Ante esto, se ha visto incrementada la preocupación internacional por combatir estos comportamientos. En este contexto, el Consejo de Europa, institución supranacional, pionera en materia deportiva, aprobó en 2014 el Convenio sobre Manipulación de Competiciones Deportivas, que contiene políticas educativas y de concienciación, medidas preventivas y de cooperación, así como anima a los Estados a criminalizar determinados comportamientos ilícitos en el deporte.

1 Artículo entregado por el autor el 15 de marzo de 2019 y aprobado el 25 de junio de 2019

2 Catedrático de Derecho Internacional de la Universidad de Salamanca, España. orcid.org/0000-0001-5619-1086 
Rev. CAP Jurídica Central N. 4 - agosto 2019, ISSN 2550-6595

\section{PALABRAS CLAVE:}

Consejo de Europa, deporte, manipulación de competiciones, España, Tribunal de Justicia de la Unión Europea.

\section{ABSTRACT:}

In the last years, sports have increased their economic dimension, being considered not only a healthy social practice. This fact has contributed for the appearance of a new criminality, based on fraud and corruption, linked to manipulation of sports competitions and sports bettings. Because of that, international concern for fighting against these behaviors has been strengthened. In this context, the Council of Europe, a supranational pioneering institution in sports area, approved the Convention on Manipulation of Sports Competitions in 2014. This text contains educational and awareness-raising policies, preventive and cooperative measures, and encourages each Party to criminalise several illicit acts in sports.

\section{KEYWORDS:}

Council of Europe, sports, manipulation of sports competitions, Spain, European Union Court of Justice. 


\section{Introducción}

El deporte, en sus múltiples manifestaciones y disciplinas, se ha convertido, en los últimos tiempos, en una de las actividades sociales con mayor arraigo y capacidad de movilización. Se revela, además, como un elemento fundamental del sistema educativo y su práctica es importante en el mantenimiento de la salud ${ }^{3}$. Del mismo modo, como realidad colectiva, éste ha alcanzado unas dimensiones sociológicas, económicas, culturales y educativas que aportan valores -como el espíritu de equipo, la solidaridad, la tolerancia y el juego limpio- y modelos de comportamiento que traspasan todo tipo de límites y fronteras territoriales, sociales y económicas ${ }^{4}$. Ello explica que, en la actualidad, hablar de deporte signifique referirse a un fenómeno social de gran envergadura, de potente proyección mediática y de una internacionalización al alcance de pocos otros movimientos culturales y sociales ${ }^{5} \mathrm{o}$, incluso, económicos.

Con todo, a la vertiente social, se ha añadido una crecientemente relevante dimensión económica, que ha convertido a las distintas modalidades deportivas en un negocio que ha sido objeto de atracción de determinadas prácticas indeseables ${ }^{6}$, en la búsqueda de obtener un lucro personal utilizando el artificio, el engaño y la

3 De Vicente Martínez. Derecho Penal del Deporte. 2010, p.13.

4 Benítez Ortúzar. El delito de "fraudes deportivos". Aspectos criminológicos, político-criminales y dogmáticos del artículo $286 b i s .4$ del Código Penal. 2011, p. 15.

5 Morillas Cueva. Derecho y deporte. Las múltiples formas del fraude en el deporte. 2017, p. 5.

6 De Vicente Martínez. Derecho Penal del Deporte ...op. cit., p. 485. 
manipulación ${ }^{7}$. Por todo ello, puede decirse que el fenómeno del deporte es, hoy, transversal y se ve confrontado a nuevos retos y amenazas, tales como la presión comercial, la explotación de jóvenes jugadores, el dopaje, el racismo, la violencia, la corrupción o el blanqueo de dinero $^{8}$. La adulteración de resultados ha comenzado a verse como un grave problema que, con carácter transnacional, se está manifestando con mucha virulencia, en gran medida propiciado por la globalización del juego por internet y las apuestas, que llegan a suponer una gran amenaza mundial para la integridad del deporte ${ }^{9}$. Precisamente este elemento transfronterizo de la corrupción motiva la creciente preocupación de las instituciones y organismos supranacionales por la prevención y la lucha eficaz contra ella, tarea que requiere una estrecha cooperación internacional ${ }^{10}$.

En este sentido y en primer lugar, pueden destacarse, en el contexto de la UNESCO, algunas de las medidas propuestas en los distintos textos de la Conferencia Internacional de Ministros y Altos Cargos encargados de la Educación Física y el Deporte (MINEPS).

Así, en la Declaración de Berlín, de 30 de mayo de 2013, (MINEPS V) y, en concreto, en la Comisión III, dedicada a la preservación de la integridad en el deporte, se reconoce ya que la integridad del deporte se ve amenazada por el dopaje en el

7 García Silvero. Iniciativas para la reforma del deporte profesional y la represión del fraude deportivo en España. Capítulo 6. 2009, p. 145.

8 Comisión Europea. Libro Blanco sobre el Deporte. 2007, p. 2.

9 Andrés Alvez. Análisis del Convenio del Consejo de Europa sobre la Manipulación de las Competiciones Deportivas. 2014, pp. 101-102.

10 Morillas Cueva. Derecho y deporte. Las múltiples formas del fraude en el deporte ...op. cit., p. 4. 
deporte, la manipulación de las competiciones deportivas y las prácticas corruptas en los planos local, nacional, regional $e$ internacional y se conmina a los Estados miembros de la UNESCO a adoptar una serie de políticas, entre las que caben destacarse: el compromiso a conceder la importancia debida y financiación a la investigación de las actividades delictivas que se producen en el ámbito del deporte; la garantía de que las autoridades de seguridad y jurídicas dispongan de la capacidad operativa adecuada para combatir la manipulación de las competiciones deportivas; la posibilidad de instauración de sanciones penales que sirvan como factor disuasorio contra la manipulación de las competiciones deportivas y contra el dopaje en el deporte o el establecimiento de organismos reguladores de las apuestas que mantengan una comunicación fluida con las autoridades de seguridad y las organizaciones deportivas para intercambiar información y proporcionar educación preventivall.

La misma línea ha seguido la Conferencia de Kazán, celebrada entre el 13 y el 15 de julio de 2017, (MINEPS VI), la cual en su Plan de Acción conecta el deporte con la Agenda 2030 y los Objetivos de Desarrollo Sostenible e incluye en su política principal III proteger la integridad en el deporte y, en específico en lo que aquí nos interesa: la directriz III.4, la cual busca fortalecer las medidas contra la manipulación de las competiciones deportivas; dicho objetivo hará necesario adoptar medidas legislativas y de aplicación de la ley concretas y forjando alianzas entre las administraciones públicas, las organizaciones deportivas y los operadores de apuestas, así como potenciar la cooperación internacional $^{12}$.

11 UNESCO. Declaración de Berlín. MINEPS v. 2013, pp. 12-16.

12 UNESCO. Plan de Acción de Kazán. MINEPS VI. 2017, p. 6 y 13. 
En un segundo lugar, más allá de documentos programáticos, la primera institución supranacional en aprobar una norma concreta en la lucha contra el fraude deportivo ha sido el Consejo de Europa, organización internacional que tiene como objetivo principal la defensa y protección de la democracia, el Estado de Derecho y los derechos humanos, en particular los civiles y políticos. El Consejo de Europa aglutina prácticamente a la mayoría de Estados europeos -todos salvo Bielorrusia- y suma algunos otros geográficamente asiáticos -como Armenia, Azerbaiyán o Georgiay del continente europeo -Groenlandia-. Como indicamos, esta organización ha sido pionera en responder normativamente a la corrupción en eventos deportivos, adoptando, en el 2014, el Convenio número 215, sobre Manipulación de Competiciones Deportivas, que comentaremos a continuación, y que nació con la intención de ofrecer una respuesta global a este problema ${ }^{13}$.

13 Andrés Alvez. Análisis del Convenio del Consejo de Europa ...op. cit., pp. 101-102. 


\section{El Consejo de Europa y el deporte}

El Convenio sobre Manipulación de Competiciones Deportivas -en adelante, el Convenio sobre Manipulación de Competiciones o, simplemente, el Convenio- no es el primer esfuerzo del Consejo de Europa en la tutela del hecho deportivo. Si bien el deporte no aparece como un ámbito específico de promoción de la democracia, los derechos humanos y el Estado de Derecho en el Convenio de Londres, de 5 de mayo de 1949, fundacional de esta organización supranacional, se identificó tempranamente esta esfera como una actividad capaz de fortalecer la cohesión social, la cultura y la educación, en el seno de los países miembro del Consejo de Europa ${ }^{14}$.

Así, el deporte se incluyó en el ámbito institucional del Consejo de Europa en 1976, con la creación del Comité Directivo para el Desarrollo del Deporte -conocido por sus siglas, (CDDS) encargado, fundamentalmente, de promover los valores de la organización a través del deporte. Un paso posterior, en el contexto de la organización supranacional, fue la puesta en marcha de un foro específico dirigido a fomentar la cooperación entre los Estados miembro en la esfera deportiva. Así, el 11 de mayo de 2007, el Comité de Ministros adoptó el Acuerdo Parcial Ampliado sobre Deporte (EPAS), por sus siglas en inglés-, en la búsqueda de preservar la independencia y la autorregulación en el deporte y en la lucha contra ciertas conductas ilícitas, como el dopaje o la violencia, especialmente en el fútbol ${ }^{15}$.

\footnotetext{
14 Pérez GonzÁlez. A propósito de la acción del Consejo de Europa en el ámbito del deporte: análisis del Convenio europeo sobre la Manipulación de Competiciones Deportivas. 2015, p.76.

CONSEJO DE EUROPA. Acuerdo Parcial Ampliado sobre Deporte.
} 
Desde el punto de vista sustantivo, destacan, en un primer momento, la Carta Europea del Deporte para Todos, adoptada en 1975, sin valor jurídico vinculante, que supone un primer eslabón en los esfuerzos del Consejo de Europa en el contexto deportivo. Se declara en este documento que todos tienen derecho a participar en actividades deportivas e identifica al deporte como un elemento de desarrollo sociocultural ${ }^{16}$. Supuso el preludio del segundo gran documento en esta materia: la Carta Europea del Deporte, adoptada en 1992 y revisada en 2001, cuyos principios básicos, enunciados en el artículo 1, se centran en la promoción de la educación física y la práctica del deporte ${ }^{17}$.

Para finalizar estos comentarios preliminares, se ha de señalar que el Convenio que vamos a analizar no es el único instrumento normativo emanado del Consejo de Europa que busca luchar contra lo que se ha dado en llamar las lacras del deporte ${ }^{18}$. Esta institución supranacional ha luchado contra los aspectos negativos en el deporte a través de tres normativas supranacionales: el Convenio sobre Seguridad integrada y enfoque en seguridad y servicios en partidos de fútbol y otros eventos deportivos (Convenio N. 218 del Consejo de Europa), abierto a la firma en 2016, aunque todavía no en vigor -y que viene a sustituir al Convenio sobre Violencia e irrupción de Espectadores, N. ${ }^{\circ} 120$ del Consejo de Europa-, el Convenio contra el Dopaje de 1989, en vigor desde el 1 de marzo de 1990 (Convenio N. ${ }^{\circ}$ 135) y el texto que vamos a estudiar a continuación, el Convenio sobre

16 Pérez González. A propósito de la acción del Consejo de Europa ...op. cit., p. 78

17 UNESCO. Carta Europea del Deporte. 1992.

18 Pérez GonZÁlez. A propósito de la acción del Consejo de Europa ...op. cit., p. 79. 
INICIATIVAS SUPRANACIONALES ANTE LA CORRUPCIÓN EN EL DEPORTE Y SU INCIDENCIA EN EL JAVIER SÁNCHEZ BERNAL ORDENAMIENTO JURÍDICO ESPAÑOL:

Manipulación de Competiciones Deportivas (Convenio N. 215 del Consejo de Europa). 


\section{El Convenio sobre Manipulación de Competiciones Deportivas}

Como venimos diciendo, este Convenio viene a suponer el tercero de los pilares de actuación del Consejo de Europa en la esfera deportiva. Fue adoptado en Magglingen-Macolin, Suiza, el 18 de noviembre de 2014. Según establece su artículo 32.4, el texto entrará en vigor el primer día del mes siguiente a la expiración de un plazo de tres meses a partir de la fecha en que cinco Estados que lo hubieran firmado -entre ellos, al menos tres han de ser miembros del Consejo de Europa- hayan expresado su consentimiento en quedar obligados por el Convenio. A fecha de redacción de este trabajo, el Convenio ha sido firmado por 32 Estados miembro del Consejo de Europa, pero solo ratificado por tres: Noruega, Portugal y Ucrania, por lo que el texto normativo aún no se encuentra en vigor, al no contarse con la voluntad de cinco Estados en quedar obligados por el mismo. España, por su parte, firmó el texto el 7 de julio de 2015, no habiendo procedido, todavía, a su ratificación.

Además, tal y como se apuntará brevemente al final de este texto, la propia eficacia del Convenio ha sido puesta en tela de juicio, por cuanto días antes de la adopción del Convenio, Malta solicitó un Dictamen al Tribunal de Justicia de la Unión Europea -en adelante, TJUE- sobre la compatibilidad de la norma con determinadas disposiciones del Tratado de Funcionamiento de la Unión Europea -en adelante, TFUE-, en particular en lo que respecta a las apuestas deportivas ilegales en relación con algunas reglas fundamentales del mercado único, viéndose comprometida la eficacia, concretamente, de los artículos 18, 49 y 56 del Convenio. Si nuestro estudio es acertado, a fecha de elaboración de esta investigación dicho 
Dictamen N. ${ }^{\circ}$ 1/2014 no ha sido aun elaborado por el referido Tribunal.

En todo caso, el objetivo de las siguientes líneas es examinar el contenido del texto normativo, incidiendo en las bondades y avances que supondría consentir en obligarse por las disposiciones del Convenio, como mecanismo de lucha contra la adulteración fraudulenta de resultados deportivos.

\section{1. Estructura y propósitos del Convenio}

El Convenio está formado por un Preámbulo y 41 artículos, distribuidos en 9 capítulos. El primero de estos capítulos -artículos 1 al 3-contiene la finalidad, los principios rectores que inspiran el texto y algunas definiciones relevantes. El segundo -artículos 4 al 11- recoge medidas de prevención y cooperación entre los Estados, además de otras medidas. El tercero, por su parte -artículos 12 al 14-, prevé herramientas de intercambio de información entre autoridades. El capítulo IV -artículos 15 al 18contiene normas de derecho penal sustantivo y de cooperación en la vigilancia de su cumplimiento. El quinto, formado por los artículos 19 al 21, contiene una serie de medidas de carácter procesal, centradas en la competencia, el procedimiento penal y medidas de vigilancia del cumplimiento de la ley. El capítulo VI -artículos 22 al 25- se destina a las sanciones y las medidas de decomiso. El séptimo capítulo, integrado por los capítulos 26, 27 y 28, recoge herramientas de cooperación internacional en materia judicial y en otros ámbitos. El penúltimo, a su vez -formado por los artículos 29 al 31- contiene instrumentos de seguimiento del propio Convenio. Por último, el capítulo IX -artículos 32 al 41- se dedica a las disposiciones finales. 
La razón de ser de este instrumento jurídico, expresada en el Preámbulo, reconoce el carácter transnacional y los vínculos de la criminalidad basada en la manipulación de competiciones deportivas con la delincuencia organizada, amén de considerarla una amenaza global a la integridad del deporte. La eficacia de la lucha contra estos comportamientos ilícitos pasa, para los redactores del texto, por conseguir una cooperación nacional e internacional más intensa, rápida, sostenible y que funcione correctamente.

Por su parte, la finalidad declarada del Convenio, referida en su artículo 1, es combatir la manipulación de las competiciones deportivas a fin de proteger la integridad y la ética deportivas respetando la autonomía del deporte. Para ello, se enumeran una serie de metas que deben alcanzarse respetando siempre los derechos humanos, y los principios de legalidad, proporcionalidad y protección de la vida privada y de los datos personales -artículo 2-. Estos objetivos específicos son los siguientes:

- Prevenir, detectar y sancionar la manipulación nacional o transnacional de las competiciones deportivas nacionales y transnacionales.

- Promover la cooperación nacional e internacional contra la manipulación de competiciones deportivas entre las autoridades públicas competentes, así como con las organizaciones y actores que participen en las competiciones y en el ámbito de las apuestas deportivas.

Los sujetos de referencia para este instrumento normativo -artículo 3.6-; las personas físicas y/o jurídicas a las que va dirigido el mismo son: los deportistas, su personal de apoyo 
-entre los que menciona el Convenio a los entrenadores, directores deportivos, agentes, personal del equipo, personal médico o paramédico y cualquier otro responsable o persona que trabaje con los deportistas-; propietarios, accionistas, directivos o cualquier otro responsable de las entidades deportivas, árbitros, jueces o cualquier otra persona acreditada; así como al personal de las organizaciones deportivas nacionales e internacionales, que según la legislación correspondiente tuvieran en cada ámbito de competencia y modalidad deportiva ${ }^{19}$.

\section{2. Contenido del Convenio}

A lo largo del articulado, el instrumento jurídico en estudio establece una serie de recomendaciones y obligaciones a los Estados parte, en aras a un combate eficaz contra la manipulación de competiciones deportivas. En efecto, el Convenio identifica la coordinación y cooperación entre todos los agentes involucrados en la práctica deportiva como una de las claves para conseguir los objetivos propuestos ${ }^{20}$. Para conseguirlo, cada Estado coordinará las políticas y actuaciones de las autoridades públicas competentes para luchar contra la adulteración de resultados y alentará a las organizaciones deportivas, organizadores de competiciones y operadores de apuestas deportivas a implementar, cuando sea oportuno, las disposiciones del propio Convenio -artículo 4-.

19 Andrés Alvez. Análisis del Convenio del Consejo de Europa ...op. cit., p. 104.

20 Pérez GonzÁlez. A propósito de la acción del Consejo de Europa .... CIT., p. 82. 
Rev. CAP Jurídica Central N. 4 - agosto 2019, ISSN 2550-6595

\section{A) Obligaciones de cooperación y prevención}

En primer término, se imponen una serie de obligaciones, en materia de prevención y coordinación a los diversos agentes involucrados en la práctica deportiva. A los Estados, en cooperación con el resto de organizaciones e instituciones deportivas, se les encomienda la tarea de valorar los riesgos inherentes a las prácticas de manipulación de competiciones $\mathrm{y}$, en su caso, adoptar procedimientos y reglamentos que permitan combatir la manipulación de eventos deportivos -artículo 5-; todo ello, acompañado de medidas que fomenten la concienciación, educación, formación e investigación en este campo -artículo 6-.

Los Estados serán, también, los encargados de adoptar medidas legislativas y de cualquier otra índole, pertinentes para garantizar la transparencia de la financiación de las organizaciones e instituciones deportivas a las que apoyen económicamente, colaborar en el combate de estos frente a la manipulación de competiciones y retirar dicho apoyo económico, en caso de que las instituciones deportivas no apliquen finalmente las normas anti manipulación.

El artículo 7, por su parte, contiene una serie de medidas, basadas en el buen gobierno deportivo, a ser aplicadas por las organizaciones deportivas y los organizadores de competiciones. Así, se exhorta a prevenir los conflictos de intereses -prohibiendo a los operadores deportivos a que apuesten en los eventos deportivos en los que participen y prohibiendo el uso y difusión de información privilegiada-, a cumplir las obligaciones contractuales y de cualquier otra naturaleza pertinentes, a informar sobre cualquier actividad, incentivo o contacto sospechoso para 
poder vulnerar las normas anti manipulación. Del mismo modo, se pide de las organizaciones deportivas y de los organizadores de competiciones que apliquen sistemas eficaces de supervisión y de información, ante posibles vulneraciones de las normas anti manipulación y, en su caso, que impongan sanciones y medidas disciplinarias específicas, efectivas, proporcionadas y disuasorias. El último inciso de dicho precepto dispone la compatibilidad de la responsabilidad disciplinaria deportiva con las eventuales sanciones penales, civiles o de carácter administrativo; principio de compatibilidad también cristalizado en nuestra legislación deportiva, en concreto, en el artículo 5 del Real Decreto 1591/1992, sobre Disciplina Deportiva.

El ámbito de las apuestas deportivas ocupa un lugar preeminente en el Convenio. El artículo 9 impone a los Estados la necesidad de designar una autoridad regulatoria de las apuestas deportivas, quien ostente potestades de información, cooperación, regulación del ámbito de las apuestas y alerta ante actividades sospechosas. Respecto de los operadores de apuestas deportivas -artículo 10-, el Convenio busca evitar los conflictos de intereses, prohibiendo que los operadores de apuestas puedan participar apostando con los productos que ofrecen y evitando que la posición de patrocinador o co-propietario del operador de apuestas, respecto de una organización deportiva, pueda facilitar la adulteración de una competición, así como el uso ilegítimo de información privilegiada.

Se trata de involucrar a las organizaciones e instituciones deportivas en la tarea de las autoridades del juego de concienciar a propietarios y trabajadores sobre las consecuencias negativas de manipular una competición y de la forma de combatirla y, del mismo modo, de informar, sin dilación a las autoridades, respecto de cualquier apuesta irregular o sospechosa. 
Especialmente interesante resulta el artículo 11 del instrumento normativo. Éste se dedica a prever diversos medios para combatir las apuestas deportivas ilegales, como medio para evitar la manipulación de las competiciones.

Algunas de las medidas que se sugieren en el propio texto son las siguientes:

- Cierre o limitación directa o indirecta del acceso a los operadores de apuestas deportivas remotas, y cierre de los operadores ilegales de operadores de apuestas offline situados en el territorio de un Estado parte;

- Bloqueo de los flujos económicos entre los operadores de apuestas deportivas ilegales y los consumidores;

- Prohibición de publicidad de los operadores de apuestas ilegales; $y$,

- Fomento de la concienciación de los consumidores acerca de los riesgos y consecuencias de las apuestas deportivas ilegales.

España ya ha avanzado terreno en este sentido, singularmente desde la entrada en vigor de la Ley 13/2011, de 27 de mayo, de Regulación del juego y de su normativa de desarrollo, conformada por los Reales Decretos 1613 y 1614/2011, de 14 de noviembre, que desarrollan esta ley en lo relativo a los requisitos técnicos de las actividades de juego y a las licencias, autorizaciones y registros del juego, respectivamente. Destaca la labor de la autoridad regulatoria a nivel estatal, la Dirección General de Ordenación del Juego, dependiente del Ministerio de Hacienda 
y Función Pública, así como de las Consejerías y Direcciones Generales de las Comunidades Autónomas, con competencias en materia de juego online y offline ${ }^{21}$.

\section{B) Obligaciones de intercambio de información}

Losartículos 12 al 14configuranuna serie deobligacionescentradas en el intercambio de información entre autoridades públicas, organizaciones deportivas, organizadores de competiciones, operadores de apuestas deportivas y plataformas nacionales de los distintos Estados parte. Con este fin, el artículo 13 impone designar una plataforma nacional contra la manipulación de competiciones, cuyas funciones principales vienen enunciadas en el artículo 13.1: servir como centro de información; coordinar la lucha contra la manipulación de competiciones deportivas; recibir, centralizar y analizar información sobre apuestas irregulares y sospechosas; alertar sobre incumplimientos de las previsiones del Convenio y de la normativa deportiva, y cooperar con otras organizaciones y autoridades, a nivel nacional e internacional, y con otras plataformas nacionales. Todo esto debe hacerse, según prescribe el artículo 14, garantizando la protección de los datos personales de todos los afectados.

\section{C) Normas sobre Derecho penal sustantivo}

El artículo 15 insta a los Estados parte a imponer sanciones penales a la manipulación de competiciones deportivas cometida por medio de prácticas coercitivas, corruptas o fraudulentas, de acuerdo al Derecho interno de los Estados. El artículo 17, por su parte, exige

20 Andrés Alvez. Análisis del Convenio del Consejo de Europa ...op. cit., pp. 108-109. 
tipificar como delito la complicidad intencionada en los delitos de corrupción deportiva. España cumple con la exigencia descrita en el artículo 15 desde que la Ley Orgánica 5/2010 introdujo en el Código penal el delito de corrupción en el deporte, como un subtipo de delito de corrupción entre particulares.

El primer numeral del artículo 16 impera a los Estados a tipificar como delito las conductas de blanqueo, definidas en determinados instrumentos normativos supranacionales; en concreto, el artículo 9.1 y 2 del Convenio del Consejo de Europa relativo al Blanqueo, seguimiento, embargo y comiso de los productos del delito y a la financiación del terrorismo, el artículo 6.1 de la Convención de Naciones Unidas contra la Delincuencia Organizada Transnacional y el artículo 23.1 de la Convención de Naciones Unidas contra la Corrupción, en los términos previstos respectivamente. El apartado $3^{\circ}$ del artículo 16 de este Convenio prevé una cuestión interesante: la posibilidad de incluir la manipulación de competiciones deportivas en la normativa de prevención del blanqueo de capitales. En este sentido, el artículo 33.1 del Real Decreto 1614/2011 impone ciertas obligaciones a los operadores de apuestas, en materia de prevención de blanqueo de capitales, por ejemplo, la comunicación de operaciones sospechosas a la Comisión Nacional del Juego y al Servicio Ejecutivo de la Comisión de Prevención del Blanqueo de Capitales e Infracciones Monetarias, órgano considerado la Unidad de Inteligencia Financiera en nuestro país.

El artículo 18, por último, prescribe a los Estados que tomen las medidas legislativas necesarias para garantizar que las personas jurídicas respondan de los delitos, comprendidos en los artículos 15 al 17 del Convenio, estableciendo el consiguiente sistema de responsabilidad de dichas entidades. 


\section{D) Competencia, aspectos procesales y de ejecución}

El artículo 19.1 declara la competencia de la jurisdicción de un Estado parte respecto de los delitos mencionados en el Convenio, cometidos en su territorio, a bordo de un buque que enarbole su pabellón o de una aeronave registrada, de acuerdo a su Derecho interno o cometido por uno de sus nacionales o por una persona que resida habitualmente en su territorio. El párrafo $3 .^{\circ}$ impone la obligación de juzgar o extraditar -aut dedere aut iudicare, principio básico del Derecho internacional-, a los responsables de alguno de los delitos referidos en el Convenio, que no puedan ser extraditados a otro Estado parte por su nacionalidad.

En añadidura, el artículo 20 establece medidas de aseguramiento de las pruebas electrónicas, y el artículo 21 recomienda a los Estados a incluir medidas de protección de testigos, que puedan aportar información probatoria en un proceso abierto a un presunto responsable de los delitos referidos.

\section{E) Obligaciones en materia de sanciones}

Además de prever la necesidad de tipificar como delitos ciertas conductas, el Convenio pretende que las sanciones que se deriven de ellos sean efectivas, proporcionadas y disuasorias. El artículo 22 se dedica a las sanciones a las personas físicas, respecto de las cuales se dice que habrán de consistir en penas de privación de libertad, que puedan dar lugar a la extradición, amén de permitirse la imposición acumulada de penas pecuniarias. El siguiente precepto se dedica a las sanciones a las personas jurídicas. Tales consecuencias habrán de ser, como en el caso anterior, efectivas, proporcionadas y disuasorias, y podrán comprender la inhabilitación especial temporal o permanente del ejercicio 
de actividades comerciales, la intervención judicial e, incluso, la liquidación de la persona jurídica, en añadidura a eventuales penas pecuniarias. El artículo 24 describe determinadas sanciones administrativas, mientras que el artículo 25 impone a los Estados prever el embargo y el decomiso:

- De los bienes, documentos y otros instrumentos empleados, o que fueran a serlo, para cometer los delitos enunciados en el Convenio.

- El producto de dichos delitos, o bien el valor equivalente a dicho producto.

\section{F) Cooperación judicial internacional}

Como se ha dicho anteriormente, la cooperación es otro de los pilares de este Convenio. Se establece que los Estados parte deben cooperar entre sí, de conformidad con las disposiciones del propio instrumento normativo y de otras normas internacionales y regionales aplicables, y otros acuerdos que hayan podido adaptarse, de acuerdo a los ordenamientos internos ${ }^{22}$. En el artículo 26 se incluyen medidas de cooperación judicial internacional en materia penal, el 27 amplía las medidas de cooperación, incluidas en el capítulo II, en aspectos de prevención y, por último, el 28 se focaliza en incluir medidas de cooperación entre las distintas organizaciones deportivas internacionales.

22 Andrés Alvez. Análisis del Convenio del Consejo de Europa ...op. cit., p. 116. 


\section{G) Medidas de seguimiento del Convenio}

La herramienta jurídica en estudio apuesta por la creación de un Comité de Seguimiento para los fines previstos en la propia norma. Si bien profundizar en dicho Comité excede a los fines de este trabajo, no está demás citar que el artículo 30, se dedica a determinar las reglas de composición de dicha institución y el 31, a diseñar algunas funciones que se le encomiendan, como particularmente formular recomendaciones a los Estados parte, informar a las organizaciones internacionales y a los ciudadanos sobre actividades emprendidas al albor del Convenio, emitir dictámenes para el Comité de Ministros, entre otras.

\section{H) Disposiciones finales}

Por último, los artículos 32 al 41 contienen las llamadas Disposiciones finales, entre las que se encuentran las previsiones de entrada en vigor, efectos del Convenio y su relación con otros instrumentos internacionales, condiciones y medidas de salvaguarda, aplicación territorial, reservas, enmiendas, solución de controversias, denuncia y notificación. 


\section{El Dictamen 1/2014 del Tribunal de Justicia de la Unión Europea}

Expusimos en el segundo epígrafe de esta investigación que la futura entrada en vigor y la eficacia del Convenio sobre Manipulación de Competiciones cuenta con un obstáculo relevante. Como dijimos, días antes de la adopción del texto Malta solicitó al TJUE un Dictamen acerca de la compatibilidad del entonces proyecto de Convenio con algunas disposiciones del TFUE. Los artículos más comprometidos por el pronunciamiento del Tribunal serán el 18, el 49 y el 56, en relación con la definición de apuestas deportivas ilegales, y, en resumen, se trata de determinar el grado de libertad de los Estados miembro de la UE para regular y/o limitar los juegos de azar ${ }^{23}$. Hemos de decir, sin embargo, que si el estudio realizado ha sido acertado, hasta el momento de la preparación de este trabajo, este Dictamen, numerado como el 1/2014, no ha visto la luz, por lo que deja esta cuestión todavía sin resolver.

23 Pérez GonzÁlez. A propósito de la acción del Consejo de Eluropa ...op. cit., p. 87. 


\section{A modo de conclusión}

En estas consideraciones finales no puede dejar de ponerse de relieve la pertinencia de la adopción y ratificación del Convenio del Consejo de Europa sobre Manipulación de Competiciones Deportivas. El mayor logro de este instrumento es, sin duda, combinar en su articulado políticas de educación y sensibilización, respecto del problema de la manipulación de competiciones, así como prever herramientas de prevención, coordinación y cooperación, en distintos niveles, en el combate frente a estos comportamientos ilícitos. Se busca, asimismo, involucrar en esta tarea a todos los agentes y operadores que intervienen en el ámbito deportivo, singularmente a las autoridades públicas, organizaciones deportivas, organizadores de competiciones y operadores de apuestas deportivas, en el sentido en que son definidos en el propio texto.

Supone un hito relevante, también, la previsión de medidas de carácter penal, sustantivo y procesal, en especial, aquéllas tendientes a la tipificación del delito de manipulación de competiciones, a través del fraude y la corrupción y del blanqueo del producto de manipulaciones deportivas. Y ello, tanto para personas físicas, como jurídicas, solicitando a los Estados parte la adopción de medidas efectivas, proporcionadas y disuasorias. El mandato de incriminar las conductas de fraude y corrupción en el deporte, relacionadas con la manipulación de competiciones, debe valorarse también positivamente. En este sentido, inaugura un camino por el que no se habían adentrado otros instrumentos supranacionales sobre el combate a la corrupción, tales como la Convención de Naciones Unidas contra la Corrupción, el propio Convenio Penal sobre la Corrupción del Consejo de Europa o, en el contexto del Derecho comunitario, la Decisión Marco 2003/568/JAI. 
Por último, hemos de reconocer que las previsiones del Convenio suponen un importante paso adelante en la lucha contra la adulteración de competiciones deportivas. A pesar de ello, en la futura entrada en vigor de la norma estará llamado a jugar un papel fundamental el Dictamen 1/2014 del TJUE, solicitado por Malta, en el cual el órgano jurisdiccional habrá de valorar, si las eventuales restricciones a las reglas del mercado único de la UE, contenidas en el Convenio, están justificadas y son, por ende, aplicables; de no ser así, ni la propia UE ni los Estados miembros de ésta podrían ratificar esta norma, lo que pondría en serio entredicho su eficacia ${ }^{24}$.

24 Pérez GonzÁLez. A propósito de la acción del Consejo de Europa ...op.cit., pp. 88-89. 


\section{BIBLIOGRAFÍA}

\section{Bibliografía impresa}

ANDRÉS ALVEZ, Rafael. "Análisis del Convenio del Consejo de Europa sobre la Manipulación de las Competiciones Deportivas". RevistaJurídicadeDeporteyEntretenimiento: deportes, juegos de azar, entretenimiento y música. N. ${ }^{\circ}$ 44, Cizur Menor, Aranzadi, 2014.

BENÍTEZ ORTÚZAR, Ignacio Francisco. El delito de "fraudes deportivos". Aspectos criminológicos, políticocriminales y dogmáticos del artículo 286bis.4 del Código Penal. Madrid, Dykinson, 2011.

DE VICENTE MARTíneZ, Rosario. Derecho Penal del Deporte. Valencia, Tirant lo Blanch, 2010.

GARCÍA SILVERO, Emilio Andrés. "Capítulo 6. Iniciativas para la reforma del deporte profesional y la represión del fraude deportivo en España”. CARDENAL CARRO, Miguel, GARCÍA CABA, Miguel María y GARCÍA SILVERO, Emilio Andrés (coords.). ¿Es necesaria la represión penal para evitar los fraudes en el deporte profesional? Murcia, Laborum, 2009.

MORILlAS CUEVA, Lorenzo. "Derecho y deporte. Las múltiples formas del fraude en el deporte". MORILLAS CUEVA, L. (dir.). Respuestas jurídicas al fraude en el deporte. Madrid, Dykinson, 2017. 
Rev. CAP Jurídica Central N. 4 - agosto 2019, ISSN 2550-6595

\section{Bibliografía con soporte web}

COMISIÓN EUROPEA. Libro Blanco sobre el Deporte. Bruselas, COM, 2007, p. 391 final, <http://eur-lex.europa.eu/legalcontent/ES/TXT/PDF/?uri=CELEX:52007DC0391\&fro $\mathrm{m}=\mathrm{ES}>$ [Consulta: 21/02/2018].

CONSEJO DE EUROPA. Acuerdo Parcial Ampliado sobre Deporte. <http://www.coe.int/t/dg4/epas/about/history_ en.asp>. [Consulta: 21/02/2018].

CONSEJO DE EUROPA. Carta Europea del Deporte. 1992, $<$ https://search.coe.int/cm/Pages/result_details.aspx?Obj ectID $=09000016804 \mathrm{c} 9 \mathrm{dbb}>$. [Consulta: 21/02/2018].

CONSEJO DE EUROPA. Convenio del Consejo de Europa sobre Manipulación de Competiciones Deportivas. 2014, <https://rm.coe.int/16801cdd7e.>. [Consulta: $21 / 02 / 2018]$.

PÉREZ GONZÁLEZ, Carmen. "A propósito de la acción del Consejo de Europa en el ámbito del deporte: análisis del Convenio europeo sobre la Manipulación de Competiciones Deportivas". Eunomia. Revista en Cultura de la Legalidad. N. ${ }^{\circ}$ 8, Madrid, Universidad Carlos III, 2015, pp. 71-92, < https://e-revistas.uc3m.es/ index.php/EUNOM/article/view/2477/1364>. [Consulta: 21/02/2018].

UNESCO. Declaración de Berlín. MINEPS V. 2013, <http:// unesdoc.unesco.org/images/0022/002211/221114s.pdf> [Consulta: 21/02/2018]. 
. Plan de Acción de Kazán. MINEPS VI. 2017, <http:// unesdoc.unesco.org/images/0025/002527/252725s.pdf>. [Consulta: 21/02/2018]. 